\title{
Impulso de habilidades y capacidades de innovación y creatividad en estudiantes universitarios
}

Boosting skills and abilities of innovation and creativity in university students

\author{
Edison Becerra Molina. ${ }^{1}$
}

\begin{abstract}
.
DOI: https://doi.org/10.33262/visionariodigital.v4i3.1310

Entrepreneurship is a quality and ability that a person has to prepare a thought to do something new and innovative and / or a business project, through ideas and opportunities. Entrepreneurship is a word immensely used in the business environment, by virtue of its relationship with the creation of some innovative activity, companies, new products or innovation of existing ones.

In this part of our research, four micro-entrepreneurial processes are considered from the prospective perspective and the foundational principles that must be present transversally throughout the process, as well as the integration of these methods in the professional training of its students, based on of its institutional philosophy, background and contextualization, considering the changes in the epistemological horizons of knowledge, the new international trends in higher education, as well as the current social demands placed on Ecuadorian Universities, to guarantee the academic excellence and quality of the institution.

Therefore, it is necessary within this research work to implement a theoreticalacademic management model for the development of innovative entrepreneurship policies and programs at the Catholic University of Cuenca UCACUE, which is part of the doctoral thesis of the author of this article that contributes to the training of new entrepreneurs in different careers in correspondence with the economic and social demand of the territory.
\end{abstract}

\footnotetext{
${ }^{1}$ Universidad Católica de Cuenca, Cuenca, Ecuador, jbecerram@ucacue.edu.ec
} 
To this end, it is important to strengthen the academic management of the training of leaders and entrepreneurs at UCACUE, through the integration of curricular and extracurricular activities and programs, carried out in the Academic Units within a common institutional plan.

The purpose is to determine the institutional policies for the generation of a culture of commitment, collaboration and consensus that are articulated with the axes of teaching, research and connection during the next five-year period, which contributes to development and competitiveness, considering the institutional strategy of Research, Development, Innovation and Incubation called $(\mathrm{R}+\mathrm{D}+\mathrm{i})$.

The model generates learning spaces with members of the University community and entrepreneurial and business actors from the locality and the country; to take advantage of the institutional capacity for the management of continuous training projects in the field of innovative entrepreneurship, committed to the sustainable development of Ecuador and especially the region 6.

Keywords: Curriculum, mipyme, entrepreneurship, innovation and work.

\section{Resumen.}

El emprendimiento es una cualidad y capacidad que tiene una persona para preparar un pensamiento de hacer algo nuevo e innovador y/o un proyecto empresarial, mediante ideas y oportunidades. El emprendimiento es un vocablo inmensamente utilizado en el ambiente empresarial, en virtud de su relacionamiento con la creación de alguna actividad innovadora, empresas, nuevos productos o innovación de los ya existentes. En esta parte de nuestra investigación se consideran cuatro micro procesos de emprendimiento desde lo prospectivo y los principios fundacionales que deben estar presentes de manera transversal en todo el proceso, así como la integración de estos métodos en la formación profesional de sus estudiantes, sobre la base de su filosofía institucional, los antecedentes y la contextualización, considerando los cambios en los horizontes epistemológicos del conocimiento, las nuevas tendencias internacionales de la educación superior, así como las actuales exigencias sociales planteadas a las Universidades Ecuatorianas, para garantizar la excelencia y calidad académica de la institución.

Por lo tanto, es necesario dentro del presente trabajo investigativo la implementación de un modelo de gestión teórico-académico para el desarrollo de políticas y programas 
de emprendimiento innovador en la Universidad Católica de Cuenca UCACUE, que es parte de la tesis doctoral del autor del presente artículo, que aporte a la formación de nuevos emprendedores en las diferentes carreras en correspondencia con la demanda económico- social del territorio.

Para tal fin, es importante afianzar la gestión académica de la formación de líderes y emprendedores en la UCACUE, a través de la integración de las actividades y programas curriculares y extracurriculares, ejecutados en las Unidades Académicas dentro de un plan común institucional.

El propósito es determinar las políticas institucionales para la generación de una cultura de compromiso, colaboración y consenso que se articulan con los ejes de la docencia, la investigación y vinculación durante el próximo quinquenio, que aporte al desarrollo y la competitividad, considerando la estrategia institucional de Investigación, Desarrollo, Innovación e Incubación denominada ( $1+\mathrm{D}+\mathrm{i})$.

El modelo genera espacios de aprendizaje con los miembros de la comunidad Universitaria y los actores emprendedores y empresariales de la localidad y el país; para aprovechar la capacidad institucional para la gestión de proyectos de formación continua en el ámbito del emprendimiento innovador, comprometida con el desarrollo sostenible del Ecuador y especialmente de la región 6.

Palabras claves: Currículo, mipyme, emprendimiento, innovación y trabajo.

\section{Introducción.}

La pandemia mundial del coronavirus o COVID-19, y la consecuente emergencia sanitaria, sumado a la situación económica por la que atraviesa el país, ha traído consigo impactos significativos en la actividad de muchas empresas, bajo este argumento en la región austral y el país donde los emprendimientos han dinamizado la economía local, es transcendental incorporar y promover la investigación para desarrollar competencias de innovación y creatividad en estudiantes universitarios.

Para el fomento de la innovación y creatividad se deben tomar en cuenta los diferentes entornos en los que el individuo está inmerso, desde la familia, escuela, medio ambiente y 
organización. El desarrollo de esta habilidad debe ser visualizada desde una perspectiva multifactorial para que pueda ser más completo.

El estudio contribuye al desarrollo de la presente investigación, por cuanto considera el currículo en el cual se desarrollan los planes, programas y proyectos de una institución universitaria, además de la didáctica, y los principios más generales de la enseñanza aplicable a todas las asignaturas en su relación con los procesos y la pedagogía, es decir el saber propio de los profesores, ese saber que les permite orientar los procesos de formación de los y las estudiantes, además de la integración de los dominios sustantivos como son la docencia, la investigación y la vinculación con la sociedad, que incluye el campo del emprendimiento y la gestión empresarial, tomando como referencia los aspectos documentales y bibliográficos del estudio mencionado.

Se consideraron las características que tiene un emprendedor, así como el reconocimiento de la categoría del aliento emprendedor en el aspecto social, en tal sentido es conveniente examinar si la formación puede favorecer a que las personas tengan cualidades emprendedoras, componentes del currículo que pueden ser tomados en cuenta para el diseño del modelo de emprendimiento y el rol que al Estado le compete en este sentido y determinar si la existencia de emprendimientos incrementa la tasa de empleo.

En esta orientación, la configuración mundial, reta a nuevos paradigmas de acción. Es por ello que las instituciones educativas deben cambiar sus estrategias formativas, para alinearse a este nuevo reto cumpliendo con la función que es la formación del capital intelectual que permitirá de manera adecuada incorporarse a los jóvenes profesionales al mercado productivo en condiciones más favorables.

\section{Metodologia.}

En este apartado de la investigación corresponde examinar algunos aportes teóricos sobre emprendimiento, se utilizó para llevar a cabo el estudio, indicando el paradigma de investigación positivista, tipo de investigación diseño de campo no experimental y transeccional o transversal con apoyo documental, Según, Tamayo (2003) establece que en la investigación de campo, los datos se recogen directamente de la realidad, razón por lo que 
se les denomina primarios, lo cual, según el precitado autor su valor radica en que permite cerciorarse de las verdaderas condiciones en que se han obtenido los datos, lo cual facilita su revisión o modificación en caso de surgir dudas (p.65).

El nivel de investigación fue la investigación descriptiva, según, Arias (2016), señala que: "La investigación descriptiva consiste en la caracterización de un hecho, fenómeno, individuo o grupo, con el fin de establecer su estructura o comportamiento" (p.24).

La población estuvo estructurada por 4 sectores escogidos, corresponde en esta oportunidad referirnos al universo de la población representada por los profesores de la diferentes Unidades Académicas con actividades administrativas en la áreas de emprendimiento, investigación y vinculación con la sociedad, considerando la población se tuvo 34 docentes, mediante muestreo estratificado se determinó la muestra $\mathrm{n}=30$, considerando la varianza muestral del 0.05, el nivel de confianza representó el 95\% de confianza, el error de la muestra el 1,4\% como aceptable, considerando el tamaño de la población y varianza poblacional, la validez de contenido fue realizado mediante el juicio de tres (3) expertos con experiencia en el área de emprendimiento y metodología.

La técnica de recolección de información, como uno de los ejes principales de la investigación, fue la encuesta, se elaboró el instrumento del cuestionario, para validar la "Construcción del modelo", el mismo tomó en cuenta los criterios de factibilidad curricular (cinco dimensiones), de precisión (5 dimensiones), de utilidad (4 dimensiones), de ética (3 dimensiones), de pertinencia (4 dimensiones), y contenido (4 dimensiones), cada uno de ellos con un conjunto de dimensiones en estricta vinculación con los objetivos de investigación, con opciones de respuesta dicotómicas y observaciones abiertas que a juicio de los validadores fuesen importante para mejorar la construcción definitiva del modelo.

El diseño y validación del Modelo de Gestión Teórico- Académico para el Desarrollo de Políticas y Programas de Emprendimiento Innovador en la Universidad Católica de Cuenca, desarrollados por el autor de la presente investigación, fue validado, considerando desde el punto de vista didáctico, los indicadores que tomaron en cuenta los validadores expertos, representados por la Academia, sector Gubernamental y los Sectores Productivos. Los indicadores considerados en la validación fueron la factibilidad curricular, precisión, utilidad, ética, pertinencia y contenidos. 
En cuanto a la validez, según, Hernández, Fernández, y Baptista (2016), se ha considerado la denominada validez por expertos, el cual se refiere al grado en que un instrumento de recolección de datos mide la variable en cuestión, de acuerdo al juicio o criterio de personas calificadas, con amplios conocimientos y experiencia al respecto (p.200).

Encinas (1993), señala que una vez que se cuenta con la información debidamente validada el proceso de análisis de los datos se esquematizará en: (a) Describir el tratamiento estadístico de los datos a través de figuras, tablas, dibujos, diagramas, generados por el análisis de los datos; (b) Describir datos, valores, puntuación y distribución de frecuencia para cada ítems; (c) el diseño de investigación utilizado indica el tipo de análisis requerido de acuerdo con la operacionalización de las variables en sintonía con los objetivos del presente trabajo de investigación.

Fue importante la utilización de la herramienta estadística para el desarrollo de la investigación, recurriéndose a la estadística descriptiva. El análisis de los datos es una parte fundamental de toda investigación, en tal sentido, Hernández, Fernández, y Baptista (2016), sostiene que el procedimiento que se sigue para analizar los datos consiste en: Una vez que los datos se han codificado, transferido a una matriz, guardado en un archivo y "limpiado" los errores, el investigador procede a analizarlos utilizando un programa computacional (p.272). De allí la importancia de inspeccionar, limpiar y transformar datos con el objetivo de resaltar información útil, lo que sugiere conclusiones, y apoyo a la toma de decisiones.

Posterior a los procedimientos que condujeron a reunir datos con el propósito específico de estructurar el Modelo, se procedió a revisar los datos o respuestas para luego ser analizados. Las respuestas a los cuestionarios se recopilan de forma automática y ordenada en formularios, con figuras y datos de las respuestas en tiempo real, que fueron procesados, analizados e interpretados, considerando el uso de la triangulación, validez, confiabilidad y contrastación de los instrumentos.

\section{Fundamentación Teórica.}

El marco teórico constituye la base donde se sustentó el trabajo de investigación, representa un sistema coherente y funcional de construir un modelo de gestión teórico- académico para 
el desarrollo de políticas y programas de emprendimiento innovador en la Universidad Católica de Cuenca, que aporte al perfil profesional en las diferentes carreras en correspondencia con la demanda económico- social del territorio de la zona 6, para ello analizó la tesis doctoral de Villar (2015), titulada: Los estilos de aprendizaje y su aplicación en la formación para el emprendimiento, presentada en la Universidad de Burgos, España. La tesis tuvo como propósito incidir en el apoyo al emprendimiento, en varios de los puntos claves de la recuperación económica y social: la creación de empresas, el autoempleo, la fijación de expectativas, el aprovechamiento de recursos ociosos, el flujo monetario, la inversión y el consumo.

La palabra emprendimiento es de origen francés Entrepreneur que significa "estar listo a tomar decisiones o a iniciar algo", la definición del término emprendedor ha ido desarrollándose y actualmente reduce el subempleo y la tasa de informalidad.

Sobre la base de la teoría, emprender es perseguir la oportunidad más allá de los recursos que se controlen en la actualidad", (Stevenson, 2000).

Desde una perspectiva humanista, el emprendimiento es una forma de expresión libre que permite demostrar el valor humano como una premisa importante que no tiene por qué verse desde una apariencia secundaria, este valor humano es lo significativo, y prevalece por encima de lo capital o de lo comercial, porque se sustenta la idea de trabajo por vocación, y libertad de pensamiento y el desarrollo de proyectos, donde el emprendedor desarrolla todas sus potencialidades y talentos.

La Agenda 2030 y los Objetivos de Desarrollo Sostenible de las Naciones Unidas, (2016), dentro del objetivo $\mathrm{N}^{\circ} 4$, Educación de Calidad, que tiene como premisa garantizar una educación inclusiva, equitativa y de calidad y promover oportunidades de aprendizaje durante toda la vida para todos, al respecto en la meta del objetivo 4.4 reivindica que "De aquí a 2030, aumentar considerablemente el número de jóvenes y adultos que tienen las competencias necesarias, en particular técnicas y profesionales, para acceder al empleo, el trabajo decente y el emprendimiento", de igual forma el objetivo $\mathrm{N}^{\circ} 8$, referente al trabajo decente y crecimiento económico que tiene propósito de Promover el crecimiento económico 
sostenido, inclusivo y sostenible, el empleo pleno y productivo y el trabajo decente para todos, en la meta 8.3, plantea: "Promover políticas orientadas al desarrollo que apoyen las actividades productivas, la creación de puestos de trabajo decentes, el emprendimiento, la creatividad y la innovación, y fomentar la formalización y el crecimiento de las microempresas y las pequeñas y medianas empresas, incluso mediante el acceso a servicios financieros".

Por su parte, Rasheed (2000), expresa que la educación en emprendimiento puede afectar los atributos que tengan los individuos y puede forjar actitudes emprendedoras en ellos. Puede promover cualidades psicológicas favorables para la actividad emprendedora, tales como la auto confianza, la autoestima, la auto eficacia y la necesidad de logro. Inclusive, la educación en emprendimiento para los jóvenes, puede colaborar en evitar la generación de actitudes socialmente no deseables, como la vagancia o la delincuencia.

Pérez (2016), en relación con la temática en estudio analiza el perfil de los estudiantes en cuanto a la intención emprendedora que permitan promover nuevas iniciativas más ajustadas a titulaciones o cursos. Del mismo modo, se propone una nueva metodología de análisis del perfil emprendedor que permitirá medir determinadas pautas de forma más ágil, en el proceso formativo de los futuros emprendedores. El autor indica que los emprendedores poseen una serie de particularidades que los distinguen del resto de las personas, el emprendedor es una persona con características muy individuales, entre ellas: Compromiso total, determinación y perseverancia, capacidad para alcanzar metas, orientación a las metas y oportunidades e iniciativa y responsabilidad.

La idea es posesionar el emprendimiento al interior de las Universidades, como uno de los ejes importantes, por considerar, que la formación escolarizada no es suficiente para interactuar en el mundo actual; es necesario, además, incorporar competencias transversales y ofrecer alternativas de vida para los futuros profesionales, en un contexto donde el emprendedor pueda valorar las teorías, enfoques y prácticas que se desarrollan en el mundo académico y su relación con la sociedad, con énfasis en el mundo laboral, para poder desempeñarse en condiciones favorables y exitosas. Todo lo anterior, en correspondencia con los objetivos del presente estudio. 
El autor de la investigación, considera estas vertientes importantes para tomar en cuenta en el modelo objeto del presente estudio, por cuanto presenta una visión integradora y parte de este análisis, sumado a nuestro aporte, se consideran para el desarrollo del modelo de gestión teórico académico, con respecto al profesor, hace referencia a que éste debe fungir como facilitador o guía orientador del proceso interactivo de enseñanza-aprendizaje, no deja de ser el experto en el tema, sin embargo, el alumno en los ambientes de aprendizaje pueda ser capaz de construir la generación de conocimientos, resolver problemas, utilizar capacidades de creatividad e innovación, tener respeto por sus ideas y libertad de opinión, un ambiente que no sea fastidioso para su desarrollo y que al contrario genere motivación al estudiante.

Es necesario establecer relaciones entre educación, investigación, negocios, industria, gobierno y comunidad para lograr subsistir en el ambiente global, siendo una de las necesidades actuales de la educación en el Ecuador.

Resulta importante estudiar la relación con las Universidades. Dehter (2012), planteó en su trabajo "Responsabilidad Social de las Universidades Hispanoamericanas para la Animación de la Cultura Emprendedora Regional", que las Universidades hispanoamericanas no han logrado dar respuesta a las necesidades sociales relacionadas con el desarrollo empresarial y la generación de empleo. De igual forma, el autor sostiene la importancia de la formación de los profesionales, así mismo, destaca la relevancia de los conocimientos teóricos, sobre las características de la cultura emprendedoras, de allí que es necesario que los emprendedores reconozcan los riesgos a los que hacen frente y preparar estrategias para poder llevar a término de manera exitosa un proyecto.

Vinculado a la formación y al empleo, hay varias sugerencias e ideas; las Universidades de la Ciudad de Cuenca, están respondiendo a la demanda de formación en emprendimiento de sus estudiantes; en estos momentos hay mano de obra muy calificada que está regresando de sus estudios en el exterior y hay expectativas sobre su incorporación al mercado laboral; se requiere un trabajo más articulado con las bolsas de empleo existentes en Cuenca (Cámara de Comercio, Universidades, Ministerio de Relaciones Laborales, entre otros), las cuales vinculen las demandas de la empresa con los recién graduados, que no necesariamente es la 
gente más preparada, pero puede ser la más crítica y propositiva; hay comentarios que proponen la necesidad de prepararse en áreas que se están expandiendo.

De allí, que el desarrollo económico de la provincia del Azuay tiene muchos retos que enfrentar entre los que se pueden nombrar los siguientes retos: Contar con espacios adecuados para el desarrollo del sector productivo, la formalización de la economía, la vertebración del territorio, la incorporación de las TIC, la generación de capacidades en innovación y competitividad, la vinculación de centros de formación - empresa, valor añadido en diseño, fomento del emprendimiento, la internacionalización y ampliación de mercados, acceso a la información adecuada y actualizada, la articulación efectiva de los actores del territorio, la colaboración efectiva público - privada, el reto de construir encadenamientos; y, de capacitación al sector productivo.

Siguiendo la misma línea discursiva, la Organización Internacional del Trabajo -OIT (2016), ha desarrollado instrumentos de autoevaluación con relación a estas características para emprendedores, para ser aplicadas en entrenamientos o capacitaciones de habilidades emprendedoras, con la finalidad de que los participantes puedan autoevaluar su potencial emprendedor.

Según, el Plan Nacional de Desarrollo Toda una Vida (2017-2021) el segundo Eje: Sistema Económico Sostenible al Servicio de la Sociedad y el Ambiente, su objetivo 5: Impulsar la productividad y competitividad para el crecimiento económico sustentable de manera redistributiva y solidaria, en el objetivo 5.3, vemos como las políticas públicas de emprendimiento se relacionan con la educación superior, promoviendo la investigación, la formación, la capacitación, el desarrollo y la transferencia tecnológica, la innovación y el emprendimiento, en articulación con las necesidades sociales, para impulsar el cambio de la matriz productiva.

Ley Orgánica de Emprendimiento e Innovación, (2020), sostiene de entre sus considerandos en el artículo 2, literal e, fortalecer la interacción y sinergia entre el sistema educativo y actores públicos, privados, de economía mixta, popular y solidaria, cooperativista, asociativa, comunitaria y artesanal del sistema productivo nacional. 


\section{Resultados.}

Se refiere al procesamiento y análisis de los datos obtenidos a lo largo del proceso de investigación, considerando el uso de la triangulación, validez, confiabilidad y contrastación de los instrumentos, la tabulación e interpretación de los cuestionarios dirigidos a los docentes de las Unidades Académicas de la Universidad Católica de Cuenca, son un total de 19 ítems, que responden a (4) preguntas de gestión académica, (9) políticas de emprendimiento innovador, (6) elementos curriculares, de estas dos fueron tipo Likert, para el presente análisis se han seleccionado únicamente cinco, los más transcendentales. ¿En la formación de los estudiantes de su carrera, que opciones usted considera importantes para el desarrollo de sus habilidades que involucren el aprendizaje del espíritu emprendedor?

Figura 1. Habilidades de la cultura emprendedora.

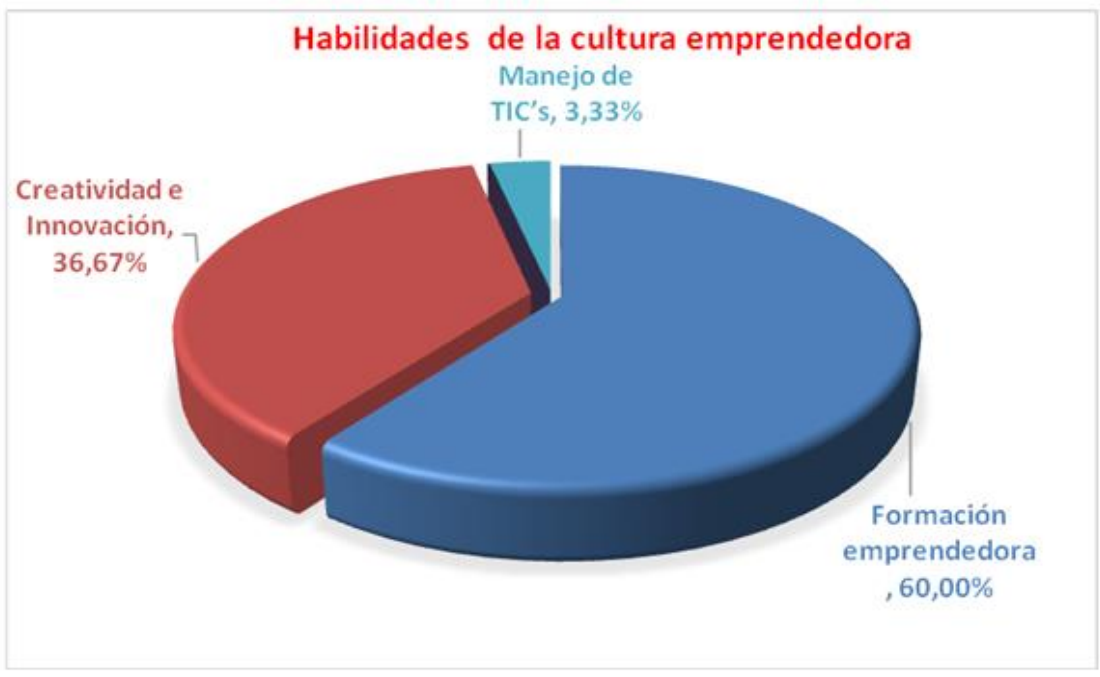

Fuente: Elaboración propia (2020).

De acuerdo a la figura $\mathrm{N}^{\circ} 1$, más del $60,00 \%$ de los docentes consideraron importante la formación emprendedora, por tales razones el emprendimiento para una persona es un desafío, debe tener una actitud positiva y una gran determinación a enfrentar retos y dificultades.

Con respecto a la creatividad e innovación, el 36,67\% sostuvieron que la creatividad y la innovación es importante para el desarrollo del espíritu emprendedor, de allí que los docentes sostienen que, como todas las actividades de la vida, el mejor método para avanzar en el 
desarrollo de una competencia o capacidad es el ejercicio constante, formar siempre será un camino acercado para perfeccionar alguna habilidad, y la creatividad.

Así mismo, el manejo de Tics, el 3.33\% de profesores encuestados, manifestaron que las Tics en la educación permiten el perfeccionamiento de competencias en el procesamiento y manejo de la información, mediante la utilización del hardware y software, por tanto el estudiante aprende a interactuar con sus compañeros y profesores, posibilitando la realización de actividades como foros, actividades, pruebas, Wikis, chat, consultas, contenido interactivos, reunión zoom y recursos como archivos, carpetas, etiquetas, libros, vídeos, entre otros.

¿Cuál de los siguientes proyectos, según su criterio se enlaza al emprendimiento considerando las tres funciones sustantivas de la educación superior, docencia, investigación y vinculación con la sociedad?

Figura 2. Proyectos que integran las funciones sustantivas (docencia, investigación y vinculación).

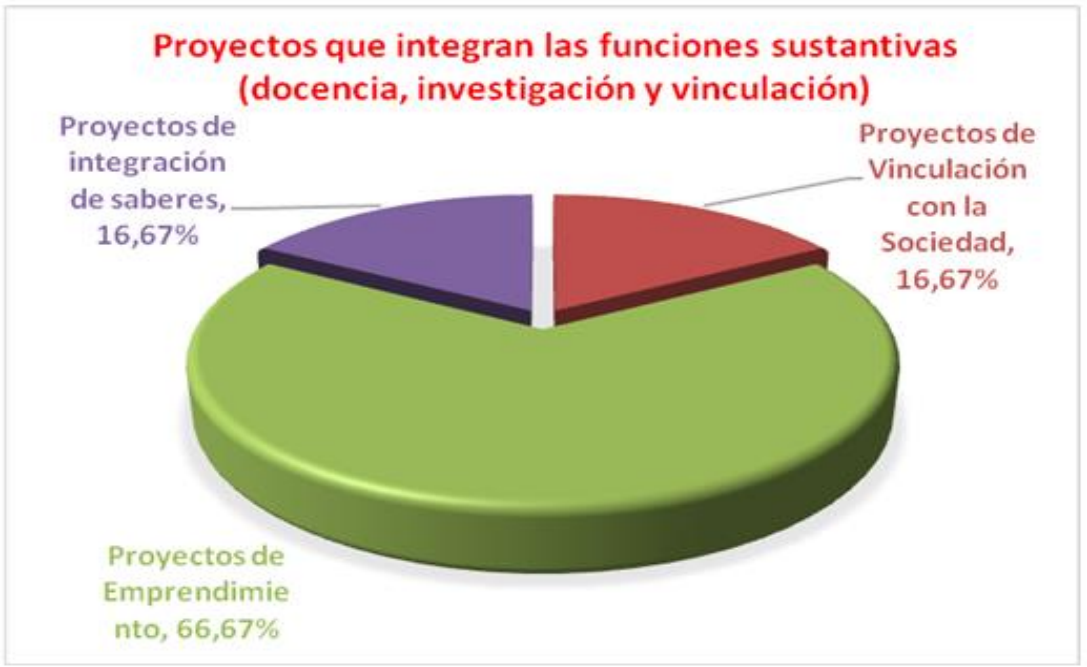

Fuente: Elaboración propia (2020).

La información recogida permitió determinar que los proyectos que integran las funciones sustantivas (docencia, investigación y vinculación), según la figura $\mathrm{N}^{\circ} 2$, son los proyectos de emprendimiento y ferias, tienen una aceptación del 66,67\%, al respecto estudiantes y docentes de las diferentes Unidades Académicas de la Universidad Católica de Cuenca en cada ciclo, ponen de manifiesto y muestran los proyectos emprendedores, de esta forma buscan promover e incentivar el desarrollo de la creación de algo nuevo e innovador, ideas 
de negocios, a través de las creatividades e innovaciones tecnológicas, para ello se hace necesario continuar, promoviendo eventos académicos extracurriculares de emprendimiento tales como el proyecto plaza, producción y ventas UCACUE, el salón de emprendimiento innovador y las visitas empresariales.

Por otro lado, el 16,67\%, los proyectos de integración de saberes fortalecen las, sustantivas (docencia, investigación y vinculación), al respecto, según la Secretaria Nacional de Educación Ciencia y Tecnología e Innovación de Ecuador -SENESCYT, el Proyecto Integrador de Saberes, (PIS), es resultado de la conjugación que debe darse entre el desarrollo de una metodología docente flexible e innovadora y la implementación de estrategias de aprendizajes significativos; básicamente se corresponde con una alternativa didácticametodológica en la cual se relacionan y ajustan los desempeños del docente y los estudiantes con las estructuras formativas generadas desde el propio proceso social, por tales razones su condición radica en la instrumentación de un sistema de trabajo colaborativo, de integración interdisciplinar, de unión de esfuerzos y participación activa en la construcción de teorías y aprendizajes desde una visión sistémica de los procesos, de allí que, facilita la construcción de saberes más complejos para la consecución de habilidades, competencias y desempeños en los estudiantes, no obstante actualmente los modelos educativos apuntan cada vez a la optimización de los aprendizajes, Wright M., Lockett A.,Claryse B. y Binks M.(2006), sostienen que en función de formar estudiantes más competentes, también es necesario previamente sistematizar teorías científicas en relación no sólo a la esencia de lo que podemos definir y entender como PIS, sino además hacia cómo orientarnos y organizarnos para su implementación y desarrollo.

Igualmente, el 16,67\% de profesores manifestaron que los proyectos de Vinculación con la Sociedad, integran las funciones sustantivas (docencia, investigación y vinculación), por tales razones promueven la transferencia e intercambio del aprendizaje entre comunidad y Universidad, acercando a los estudiantes y docentes a un contexto real a través de proyectos que generen impacto positivo especialmente en grupos vulnerables de la sociedad en la región 6 del Austro.

¿Cuáles de las siguientes capacidades, según su criterio, deben integrar la preparación específica de los profesores que imparten docencia en emprendimiento? 
Figura 3. Capacidades para la preparación específica de los profesores que imparten docencia en emprendimiento.

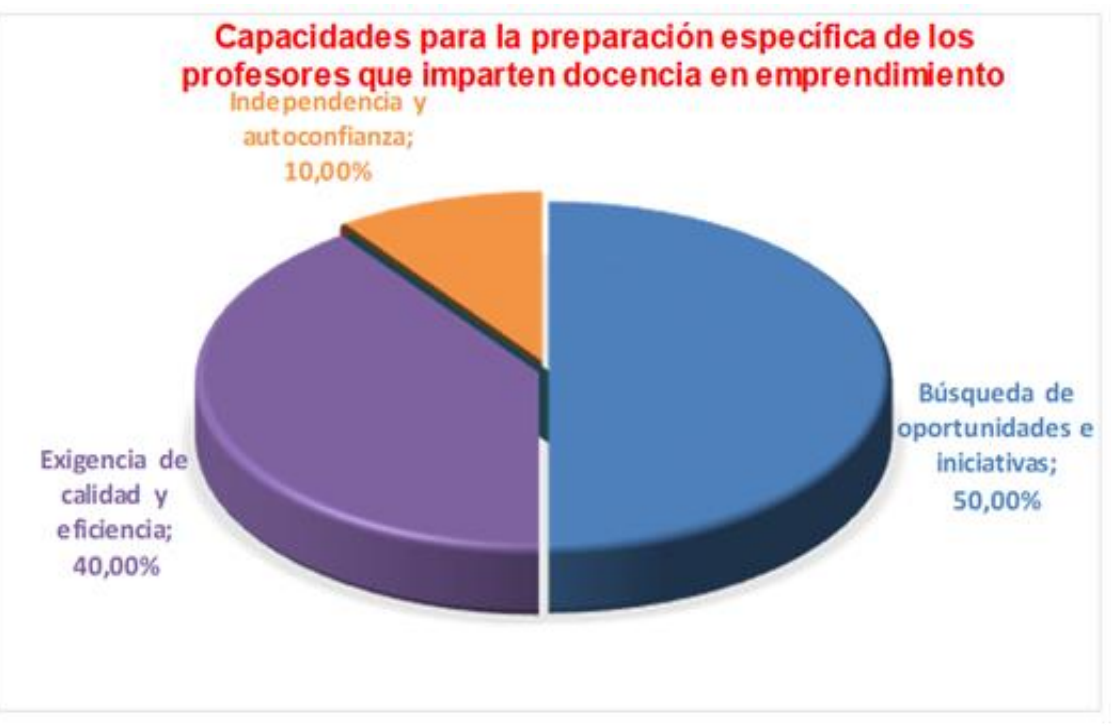

Fuente: Elaboración propia (2020).

Los resultados muestran conforme la figura $\mathrm{N}^{\circ} 3$, que es sustancial para la preparación específica de los profesores que imparten docencia en emprendimiento la búsqueda de oportunidades e iniciativas, en un $50 \%$, en este sentido los encuestados manifestaron que es importante que los profesores se capaciten en educación emprendedora, es por ello que el aprendizaje del alumnado, se produce hoy en un entorno muy dinámico y cambiante, que nos exige, tanto al profesorado como a la administración, saber conjugar los aspectos estrictamente curriculares con aquellos otros que son imprescindibles, en tal sentido los programas de cultura emprendedora tienen como objetivo generar espacios de aprendizaje que retengan los conocimientos del estudiantado, donde se pueda trabajar el currículum en torno a grandes temas significativos, donde se estimule su autonomía, iniciativa, donde la evaluación se haga también a través de las actividades diarias; siendo los docentes quienes debemos liderar el cambio educativo.

Con relación a la exigencia de calidad y eficiencia con un 40,00\% los profesores encuestados, sustentaron que los profesores que imparten docencia en emprendimiento tienen que estar preparados para afrontar las exigencias, la calidad y la eficiencia, en tal sentido se hace importante la necesidad de realizar las cosas mejores, más rápido y, si interesa, más barato. Esto tiene que ver con el entusiasmo y la necesidad de mejorar la calidad, hacer algo mejor, algo que se refleja también en la integridad de los emprendedores y en los niveles de excelencia, por lo tanto, se relaciona a la idea de perfección y a las características de una mejora continua. 
El análisis indicó, que la Independencia y autoconfianza con un 10,00\%, los profesores encuestados manifestaron que los emprendedores de éxito son personas seguras de sus capacidades, lo que les reporta serenidad, ecuanimidad y prudencia. La confianza en uno mismo significa asumir la responsabilidad total de conseguir lo que se quiere. Sobre él recaen tanto las causas del triunfo como el infortunio. Por último, tanto la persistencia, fijación de metas y la búsqueda de información no ha sido tratado por los profesores encuestados.

¿Cuáles de las siguientes opciones, según su criterio, favorecen la educación emprendedora?, (marcar 5 opciones).

Figura 4. Opciones que favorecen a la educación emprendedora.

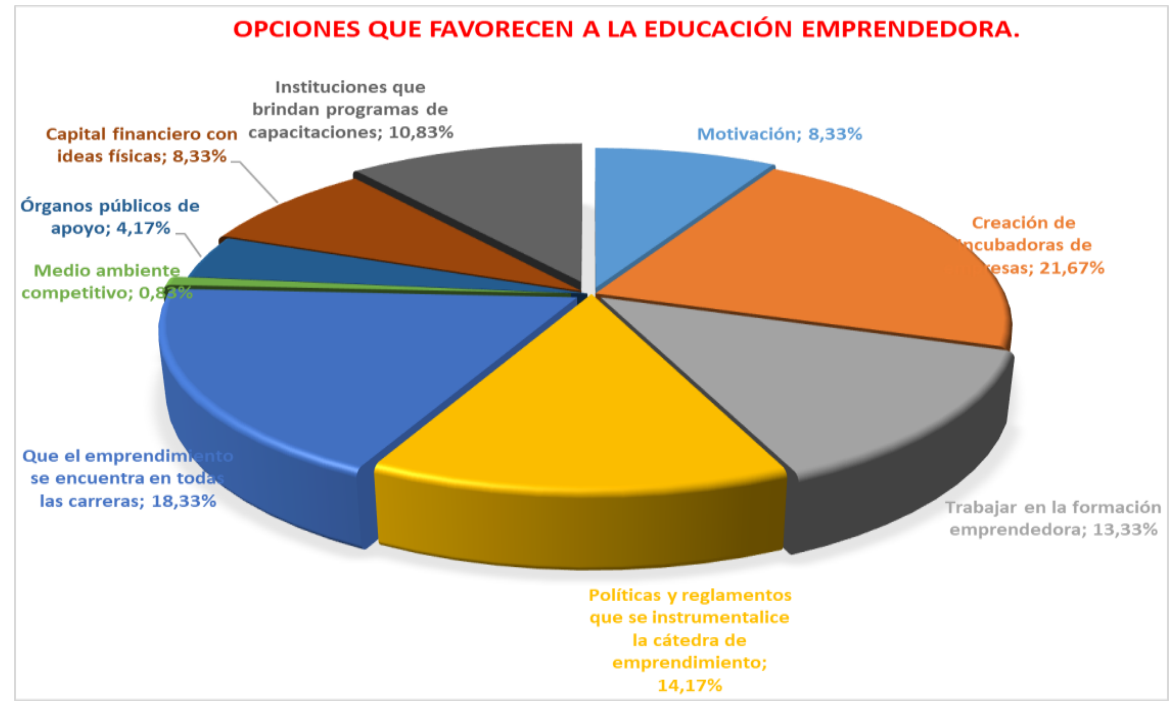

Fuente: Elaboración propia (2020).

La información recogida permitió determinar, conforme la figura $\mathrm{N}^{\circ} 4$, que las opciones que favorecen a la educación emprendedora, está la creación de incubadoras de empresas, en un 21,67\% para ello es necesario contar la cátedra de emprendimiento y la incubadora de empresas e innovación empresarial, para de esta manera promover, desarrollar y apoyar el espíritu innovador, empresarial y emprendedor de los estudiantes de la UCACUE y de la comunidad educativa, será también importante promover la creación de empresas a través de una red de servicios técnicos, gerenciales, administrativos y financieros, para de esta manera contribuir a mejorar la competitividad del tejido empresarial aportando soluciones tecnológicas que incrementen su productividad.

Con respecto a que el emprendimiento se encuentra en todas las carreras, el 18,33\% sostuvieron que es una opción importante que favorece a la educación emprendedora, de allí que el emprendimiento, debe ser considerado como un eje transversal en la formación 
profesional, ya hemos mencionado que el talento humano constituye el elemento motor de una nueva dinámica de desarrollo "desde abajo" con gente emprendedora. Su conocimiento y motivación son factores imprescindibles para esta perspectiva, por ello es necesario partir del método de enseñanza - aprendizaje propuestos en el modelo de la UCACUE, para cristalizar el éxito entonces es necesario el vínculo de la teoría con la práctica.

En relación a las políticas y reglamentos que se instrumentalice la cátedra de emprendimiento, el 14,17\%, de docentes encuestados, indicaron que se debe trabajar con mayor énfasis en temas concernientes a las leyes, políticas, reglamentaciones, normativas e incentivos para facilitar el fortalecimiento en materia de emprendimiento e innovación, para tal fin debería centrarse en el diseño e implementación de políticas institucionales de emprendimiento e innovación, así como en el intercambio de experiencias, diseño de marcos regulatorios y programas para fomentar el desarrollo e impulso del capital emprendedor.

Por su parte, trabajar en la formación emprendedora, el 13,33\%, de encuestados manifestaron que el emprendimiento se ha convertido en los últimos años en una estrategia social de formación para el desarrollo de competencias, que puede permitir a los estudiantes asumir una cultura emprendedora, realizar proyectos empresariales para el autoempleo con un mayor grado de certeza y posibilidad de mejorar la calidad de vida.

Así mismo, tenemos lo referente a las instituciones que brindan programas de capacitaciones, de lo cual el 10,83\%, de encuestados indicaron que existen instituciones que brindan programas de capacitaciones, tales como el MIPRO, Emprende Ecuador, el MIES, la Secretaria del Migrante, el Banco Nacional de Fomento y la Corporación Financiera Nacional, entre otras, receptan ideas de negocios en las distintas áreas productivas y turísticas, además que existen varios sitios virtuales que ofrece diferentes herramientas de asesoría, capacitación, consultoría, asistencia técnica y financiamiento.

Del mismo modo, la motivación que representa el 8,33\%, de los encuestados manifestaron que la motivación emprendedora, es un factor importante para contribuir con el desarrollo de una sociedad, por tales razones es transcendental el proveer instrumentos para canalizar las acciones de los emprendedores y potenciarlas para alcanzar el éxito anhelado.

De acuerdo, con el capital financiero con ideas físicas con un 8,33\%, sostuvieron que para impulsar la formación emprendedora es importante el capital financiero con ideas físicas, como es lógico para determinar la factibilidad de una inversión de idea de negocio es fundamental el análisis económico, además del financiero, mismos que consisten en un conjunto de cálculos que permiten medir la rentabilidad y analizar la viabilidad técnica y económica de un negocio. 
En cuanto, a los órganos públicos de apoyo, en un 4,17\% de los profesores encuestados manifestaron que existen instituciones como el MIPRO, MIESS, CFN, BanEcuador, Emprende Ecuador.

Por último, con respecto al Medio ambiente competitivo, el 0,83\% de docentes encuestados, indicaron que es importante un ambiente competitivo de trabajo.

¿Cuáles de los siguientes factores, según su opinión, están obstaculizando la educación emprendedora?, (marcar 5 opciones).

Figura 5. Alternativas que están obstaculizando la educación emprendedora.

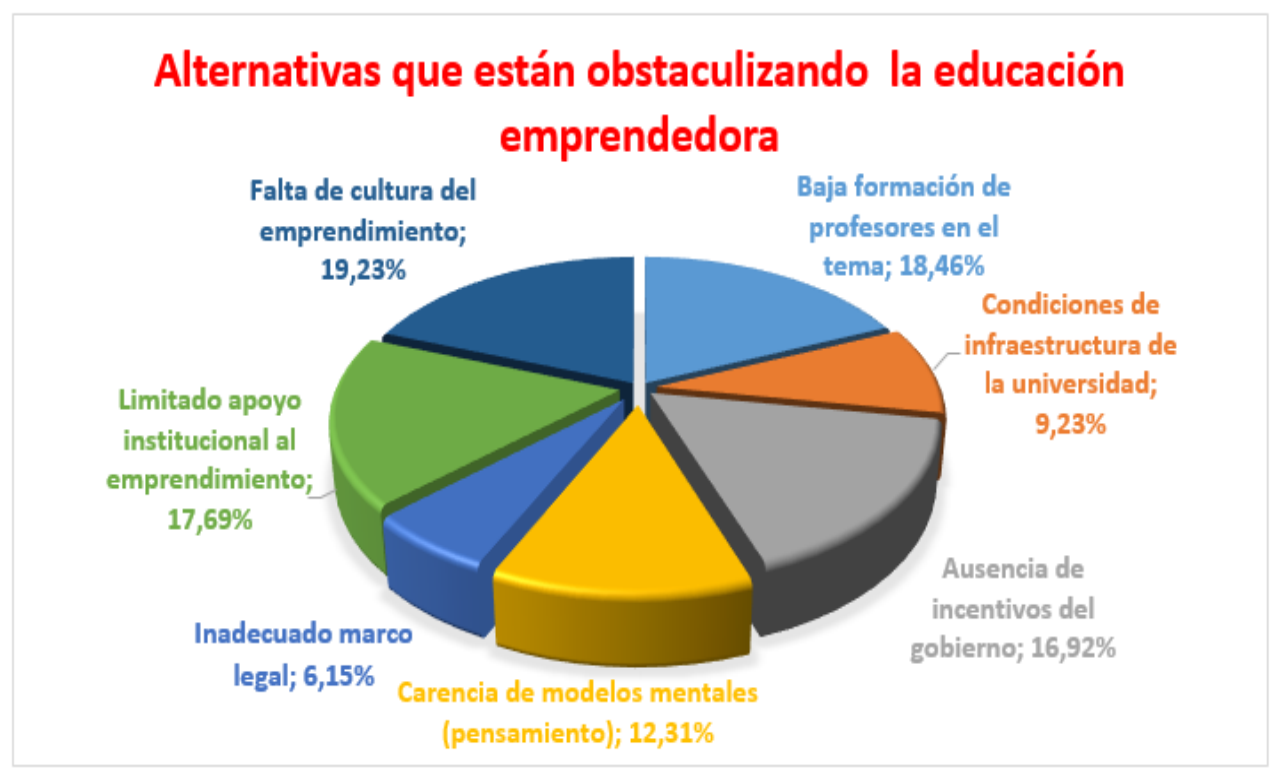

Fuente: Elaboración propia (2020).

Los resultados muestran que conforme la figura $\mathrm{N}^{\circ} 5$, los profesores encuestados pusieron de manifestaron que las alternativas que están obstaculizando la educación emprendedora en un 19,23\%, sería la falta de cultura del emprendimiento, de allí será importante apoyar a la construcción de cultura emprendedora e innovadora, especialmente al emprendimiento empresarial generador de empleo, mediante el acompañamiento a estudiantes, graduados y docentes de la Universidad Católica de Cuenca de forma significativa en la formación emprendedora, para lograr las mejores posibilidades de éxito de sus nuevas ideas de negocios y/o empresas o iniciativas empresariales.

El 18,46\%, de encuestados revelaron la baja formación de profesores, por tanto, es importante tener presente el perfil profesional del docente basado en competencias que facilita los procesos de aprendizaje del emprendimiento a los estudiantes Universitarios, los 
resultados evidencian que los docentes que tienen conocimiento en emprendimiento son facilitadores, capacitadores y comunicadores, de allí que es necesario considerar las cualidades concernientes a la capacidad de aprender, la adaptabilidad y la empatía, así mismo son responsables, respetuosos, motivadores e innovadores y emprendedores.

Un 17,69\% de los encuestados considera que hay limitado apoyo institucional al emprendimiento, siendo un factor que obstaculiza la educación, por cuanto hoy en día el emprendimiento juega un papel importante, $y$, se convierte en un tema de gran relevancia para el desarrollo de planes, programas y proyectos, cuyo propósito es crear nuevas ideas, empresas y negocios, desde esta perspectiva esto permite dinamizar y potencia la economía local regional y nacional.

De igual manera, con relación a la Ausencia de incentivos del gobierno el 16,92\% de encuestados, se pronunciaron en el hecho de que el gobierno debe impulsar el proyecto de ley orgánica de emprendimiento e innovación, esta ley tiene por objeto establecer normativa que permita al emprendedor crecer con su idea y pasar el ciclo de la persistencia, hasta lograr cristalizar los ideales y convertirse en emprendedores exitosos.

De acuerdo, con la carencia de modelos mentales (pensamiento) el 12,31\% de encuestados, indicaron que quienes quieran emprender, pero, crea que no ha aprendido lo suficiente, para poder ser emprendedores exitosos, es necesario elegir un modelo mental, mediante actividades curriculares y extracurriculares, que permitan fomentar el emprendimiento y la creación de ideas y empresas orientadas hacia la innovación.

Así mismo, con respecto a las condiciones de infraestructura de la Universidad, el 9,23\% de profesores encuestados sostuvieron, que es vital contar con aulas y espacios de aprendizaje en buen estado.

Por último, referente al inadecuado marco legal, el 6,15\%, de profesores encuestados, sostienen que es importante adecuar y actualizar el marco legal local a la realidad del sector y al nuevo estatuto de la UCACUE.

\section{Propuesta.}

Este componente apunta a generar competencias emprendedoras, por lo que su principal línea de actuación es fortalecer la Cátedra de Emprendimiento.

Las líneas de actuación se detallan de la siguiente manera:

Fortalecer la cátedra de emprendimiento y extender gradualmente su alcance de sus cursos a todas las carreras y diferenciadamente a todos los niveles. 
Insertar las clases de emprendimiento en la malla curricular de las distintas carreras a través de periodos de gestión productiva, articulados con la cátedra de emprendimiento.

Motivar el espíritu emprendedor a través del involucramiento de emprendedores y empresarios en calidad de docentes y conferencistas.

Fomentar la formación y cohesión de grupos multidisciplinarios en toda oportunidad de trabajo en equipo que se presente durante la realización de actividades académicas.

Promover el desarrollo académico, investigativo y de emprendimiento mediante el uso de tecnologías de fabricación digital y metodologías de aprender haciendo, de tal manera que permitan la creación de prototipos, productos, servicios y procesos innovadores, contribuyendo así al desarrollo emprendedor y empresarial, y para ello la Universidad Católica de Cuenca, cuenta con el Laboratorio de Fabricación Digital o FabLab.

Desarrollar una campaña de comunicación a través de medios como la radio, televisión e internet para difundir información y experiencias exitosas que motiven a la población, para ello la UCACUE cuenta con el canal Tele Cuenca y la radio Ondas Cañaris en AM y FM. Impulsar y desarrollar el alcance de la cátedra a todos los niveles significa impartir cursos de emprendimiento a alumnos y ex alumnos, de grado y posgrado, docentes e investigadores, emprendedores y empresarios.

Es importante indicar, que con mayor énfasis el emprendimiento debe ponerse de manifiesto en las carreras de la Unidad Académica de Administración que incluye Administración, Contabilidad y Auditoría; y Mercadotecnia, asimismo, se debe considerar en el modelo el resto de carreras de las Unidades Académicas, tales como Educación, Artes y Humanidades, Ciencias Sociales, periodismo, Información y Derecho, Tecnologías de la Información y la Comunicación (TIC), Ingeniería, Industria y Construcción, Agricultura, Silvicultura, Pesca y Veterinaria y Salud y Bienestar, a objeto de difundir la cultura emprendedora para el desarrollo de políticas y programas de emprendimiento innovador en la Universidad Católica de Cuenca.

Se propone a corto plazo cuatro micro procesos de emprendimiento, en el que se incluyen las actividades descritos por el investigador; los mismos que deben ser ejecutados de acuerdo a la demanda académica y de la sociedad, por las Unidades Académicas de la Matriz, Sedes y Extensiones. 
Tabla 1. Micro procesos de emprendimiento

\begin{tabular}{ccc}
\hline Micro proceso & \multicolumn{1}{c}{ Actividades } \\
\hline & $\checkmark$ Capacitación Continua de emprendimiento \\
& innovador. \\
& $\checkmark$ Eventos académicos extracurriculares de \\
& emprendimiento. \\
Fomento de cultura & $\checkmark$ Club de emprendedores. \\
emprendedora. & $\checkmark$ Sistema de apoyo a los Graduados. \\
& $\checkmark$ Difusión académica del propósito de la \\
& Formación Emprendedora: Conversatorio \\
& "Emprendimiento y el Perfil Profesional de \\
Pre-incubación de empresas. & $\checkmark$ Cátedra de Emprendimiento Innovador. \\
Incubación de empresas. & $\checkmark$ Incubación de empresas estudiantiles y \\
& desarrollo empresarial. \\
Servicios de apoyo empresarial a & $\checkmark$ Redes de apoyo al emprendimiento \\
la colectividad. & productivo e incubación: Clínica de \\
& MIPYMES.
\end{tabular}

Fuente: Elaboración propia (2020).

Estas actividades deben ser desarrolladas a través de los proyectos formulados en relación a las demandas académicas y del territorio o Zona 6 del Austro, para construir un ecosistema de emprendimiento, desarrollando conocimiento y competencias afianzando el perfil profesional. Cada Carrera de la Matriz, Sedes y Extensiones deben generar proyectos que aporten a la construcción de estos Programas y de un Plan Integrado de Emprendimiento Institucional de la Universidad Católica de Cuenca, contribuyendo al desarrollo sostenido de la Región 6. 


\section{Conclusiones.}

Se explican las principales conclusiones, integrando los resultados de las encuestas de los profesores de las diferentes Unidades Académicas de la Universidad Católica de Cuenca:

De los resultados, se evidencia que existe obstaculización emprendedora, uno de los factores que incide es el limitado apoyo institucional al emprendimiento, es decir es necesario asesorar, capacitar y promover la gestión emprendedora, la innovación, y la cultura empresarial en los estudiantes de la Universidad, para lograr las mejores posibilidades de éxito en la creación de empresas o iniciativas empresariales, generadoras de empleos y orientadas a fortalecer el desarrollo local, regional y nacional.

Otro de los factores que obstaculiza la educación emprendedora es la necesidad de mayores incentivos gubernamentales, de allí que es importante se consideren incentivos a los participantes de los distintos proyectos que se proponen, como el salón de emprendimiento innovador, proyecto plaza, producción y ventas, entre otros, mediante premios otorgados por la propia institución educativa, y actores del sector público y privado.

La información recogida, de los encuestados, profesores en el 63,29\%, sostienen que el manejo de la tecnología, el liderazgo estratégico y visionario, la iniciativa y riesgo, la persistencia y motivación, sumado a la fijación de metas y trabajo en equipo, son importantes competencias para la formación emprendedora, de allí que será importante ejecutar acciones dirigidas al desarrollo del conocimiento y la ciencia en la construcción de la cultura emprendedora, por lo tanto, es de vital importancia el manejo de la tecnología, se tiene que partir considerando las ideas de negocios, la creación de empresas o iniciativas empresariales, el desarrollo de planes de negocio, considerando la persistencia, motivación y formación de los estudiantes y docentes para la generación de emprendimientos innovadores, articulados con los procesos de educación continua en emprendimiento mediante la estructura participativa del trabajo en equipo, con el acompañamiento de tutores y mentores, creadores de empleos y orientadas a fortalecer el desarrollo local, regional y nacional.

Como es lógico hay que darle importancia a los contenidos de administración y finanzas, obligatoriamente se debe impartirlos, un emprendedor, tarde o temprano necesitará saber para entender los indicadores y exigir trabajo de sus administradores, de igual forma habrá que considerar que para que el estudiante apruebe los cursos de la cátedra de emprendimiento, debe cumplir con requisitos de rigor, debe recibir un diploma o certificado de emprendedor, independiente si terminó o no la carrera principal que está cursando.

El factor crítico de éxito del modelo de emprendimiento es lograr la participación del mayor número posible de carreras en la implementación de la cátedra de emprendimiento. La mejor 
forma de lograr esta participación es involucrando a los mejores docentes de la UCACUE para que ellos impartan los cursos de emprendimiento. En relación a los servicios de apoyo al emprendedor, que sirven para acelerar el crecimiento son los que más agregan valor a la sostenibilidad y sustentabilidad de las empresas, por lo tanto, mientras más pronto se los implemente, más rápidamente se alcanzará los objetivos propuestos.

El modelo dará lugar a buscar capital financiero con ideas físicas, para ello es necesario poner atención en la construcción y gestión de capital semilla y el fortalecimiento al emprendedor propende apoyar a los estudiantes que crean sus pequeñas unidades de producción y que buscan ganar competitividad a través de la creatividad y la innovación.

Con relación, a la concreción de proyectos de emprendimiento, la Universidad Católica de Cuenca, debería impulsar y desarrollar los ocho programas y los dieciséis proyectos, con sus distintas actividades curriculares y extracurriculares, para fomentar la cultura emprendedora en los estudiantes de las diferentes carreras de la UCACUE, con calidad y calidez, mediante la cátedra de emprendimiento, que aporte a la formación de profesionales líderes y emprendedores, y contribuya de manera sostenida a la sociedad, especialmente en el territorio.

De acuerdo a la propuesta de planificación estratégica y operativa, se describe algunas alternativas de emprendimientos que deben los estudiantes de las dieciséis carreras encuestadas llevar a cabo, ideas de negocios que han sido diseñados y formulados de acuerdo a los principios y técnicas de la investigación socioeducativa, que están sujetas a una constante revisión, mejora y ampliación de más ideas de emprendimientos, constituyéndose un modelo general que permita a la comunidad Universitaria Católica de Cuenca, integrar los demás aspectos disciplinares y profesionales dentro de cada carrera, con el fin de generar proyectos específicos que respondan a las políticas y estrategias de emprendimiento, alineados a la filosofía institucional, sus normas y reglamentos, que permita una real construcción de un Plan Integrado de Emprendimiento Institucional, con base a ser gestionado, ejecutado y evaluado.

Igualmente, es necesario que los programas, proyectos y actividades curriculares y extracurriculares propuestos para construir el plan integrado de emprendimiento, se articulen con las políticas públicas como el Plan Nacional de Desarrollo Toda una Vida, Los Planes de Desarrollo y Ordenamiento Territorial, la Agenda Zonal, y la Ley orgánica de Emprendimiento e Innovación, para la generación y desarrollo sostenible de los emprendimientos en Cuenca, para aportar a la solución de la problemática en la conducción y crecimiento de ideas de negocios y de empresas, involucradas en los planes de la Matriz productiva, con lo que se presentan nuevas oportunidades de empleabilidad. 
En relación, a la formación del personal docente que imparta las cátedras de emprendimiento e innovación, tanto estudiantes, profesionales egresados, ejecutivos empresariales y autoridades de la UCACUE, es importante que se dote a los docentes una capacitación y la actualización profesional permanente en materia de emprendimiento e innovación, a través de la implementación de instrumentos pedagógicos, tecnológicos, eficientes y eficaces, porque ello garantiza la calidad del docente para una mejor búsqueda de oportunidades e iniciativas.

La UCACUE y sus respectivas Unidades Académicas deben generar conciencia integradora, así como apoyar el proceso académico para formar profesionales competentes, con pensamiento emprendedor e innovador, con pasión y compromiso, para crear y liderar sus propias empresas de base social, economía general y base tecnológica; sobre los principios de un modelo educativo, que promueve la investigación, creatividad, innovación, productividad y competitividad.

\section{Referencias bibliográficas.}

Arias, F. (2016). El Proyecto de Investigación: Introducción a la metodología científica. (7 $7^{a}$ Edición), Caracas - Venezuela. Editorial El pasillo, C.A.

Dehter, M. 2012.Cultura Emprendedora. Programa Permanente de Capacitación para dirigentes. Impresión IDEB, Cataluña-España.

Encinas, I. (1993). Análisis de datos. Obtenido de Análisis de los datos e interpretación de los resultados, disponible en: https://www.goconqr.com/p/3298093-an-lisis-delos-datos-e-interpretaci-n-de-los-resultados-mind_maps.

Hernández, S.; Fernández, C.; Baptista, L. (2016). Metodología de la Investigación. México: Mc Graw Hill Educación, 6ta. Edición.

La Agenda 2030 y los Objetivos de Desarrollo Sostenible, (2016). Una oportunidad

para América Latina y el Caribe, Publicación de las Naciones Unidas, mayo de 2016, impreso en Santiago.

Ley Orgánica de Emprendimiento e Innovación, (2020), Esta Ley fue publicada en el Suplemento del Registro Oficial № 151, del 28 de febrero del 2020.

Organización internacional del trabajo -OIT (2016). Promoción del emprendimiento y La innovación social juvenil en América Latina. Colombia: Edición y publicación: Panamá. 
Pérez, L. (2016). Propuesta metodológica para la caracterización de pautas emprendedoras en materia de emprendimiento universitario. Validación en el Campus d'Alcoi de la Universitat Politécnica de Valencia, Recuperado de: file:///C:/Users/ZONA\%20INFORMATICA/Desktop/CAPITULO\%20II\% 2020-042018/TESIS\%20DOCTORALES- MARCO\%20TEORICO/Pérez\% 20\%20Propuesta\%20metodológica\%20para\%20la\%20caracterización\%20de\%20pautas $\% 20$ emprendedoras $\% 20$ en $\% 20$ materia\%20de\%20empr\%20ok.pdf

Rasheed, H. 2000. Developing Entrepreneutial Potencial in Youth of Entrepreneurial Education and Venture Creation. Disponible en http://www.usasbe.org/knowledge/proceedings/2001/063.pdf.

SENPLADES. (2017). Plan Nacional para el Buen Vivir 2017-2021. Quito: SENPLADES, Gaceta oficial Nº 681 del 13 de julio del 2017.

Stevenson, H. (2000). Why the entrepreneurship has won! Disponible en www.usasbe.org/pdf/cwp-2000-stevenson.pdf.

Tamayo, M. (2003). El Proceso de Investigación Científica (4ª edición). México. Editorial Limusa.

Villar, F. (2015). Los estilos de aprendizaje y su aplicación en la formación para El emprendimiento, de la Universidad de Burgos, Facultad de Educación, Burgos-España, Recuperado de: file:///C: Desktop/CAPITULO\%20II\%2020-042018/TESIS\%20DOCTORALES-MARCO\%20TEORICO/Villar_Mata \%20cREATIVIDAD\%20Y\%20EMPRENDIMIENTO\%20OK.pdf.

Wright M., Lockett A.,Claryse B. y Binks M.(2006).University spin-out companies and venture capital. Research Policy, 35, 481-501.

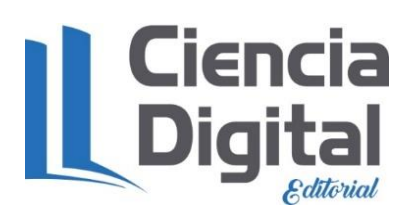




\section{PARA CITAR EL ARTÍCULO INDEXADO.}

Becerra Molina, E. (2020). Impulso de habilidades y capacidades de innovación y creatividad en estudiantes universitarios. Visionario Digital, 4(3), 122-146. https://doi.org/10.33262/visionariodigital.v4i3.1310

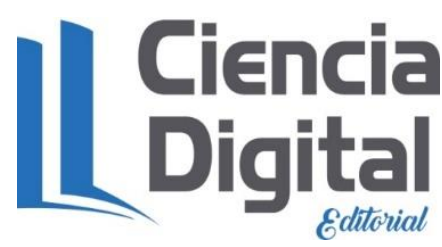

El artículo que se publica es de exclusiva responsabilidad de los autores y no necesariamente reflejan el pensamiento de la Revista Visionario Digital.

El artículo queda en propiedad de la revista y, por tanto, su publicación parcial y/o total en otro medio tiene que ser autorizado por el director de la Revista Visionario Digital.
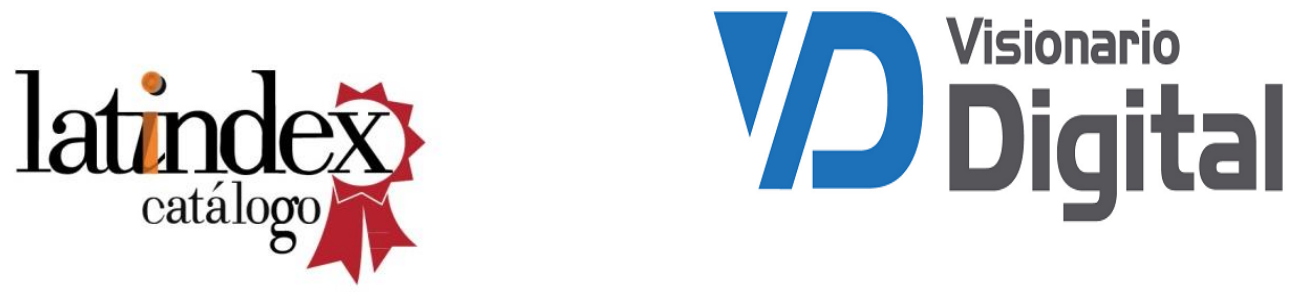